\title{
Measuring the Electrical Properties of MWNT-PA6 Reinforced Nanocomposites
}

\author{
Monica Alina Călin, ${ }^{1}$ Liliana Rozemarie Manea, ${ }^{1}$ Laurence Schacher, ${ }^{2}$ \\ Dominique Adolphe, ${ }^{2}$ Ana Lăcrămioara Leon, ${ }^{1}$ Georgeta Lidia Potop, ${ }^{1}$ and Maricel Agop ${ }^{3}$ \\ ${ }^{1}$ Faculty of Textile-Leather Engineering and Industrial Management, Technical "Gheorghe Asachi”" University of Iaşi, \\ Bulevardul D. Mangeron No. 67, 700050 Iași, Romania \\ ${ }^{2}$ Laboratoire de Physique et Mécanique Textiles EAC 7189 CNRS/UHA, ENSISA, 11 rue Alfred Werner, 68093 Mulhouse Cedex, France \\ ${ }^{3}$ Department of Physics, Technical "Gheorghe Asachi” University of Iaşi, Bulevardul D. Mangeron No. 63, 700050 Iaşi, Romania
}

Correspondence should be addressed to Monica Alina Călin; ma.calin@yahoo.com

Received 17 October 2014; Accepted 25 December 2014

Academic Editor: Davood D. Ganji

Copyright (C) 2015 Monica Alina Călin et al. This is an open access article distributed under the Creative Commons Attribution License, which permits unrestricted use, distribution, and reproduction in any medium, provided the original work is properly cited.

\begin{abstract}
The paper studies the electrical properties of polyamide 6- (PA6-) carbon nanotubes (CNTs) nanowebs, obtained through electrospinning. Three different treatments (chemical, mechanical, and mixed) were applied to the CNT in order to prepare the electrospinning solutions. For each treatment, the CNT content was different: $0.5 \%, 1 \%, 1.5 \%$, and $2 \%$. The electrical volume and surface conductivity of the obtained samples were studied by measuring their electrical volume and surface resistance. Homemade plate electrodes were used. The samples were also analyzed using a scanning electron microscope (SEM) and an atomic force microscope (AFM). Defects were found on the extremities: solvent traces, flat fibers, and beads. The mixed treatment seems too aggressive and it is not recommended. The AFM analysis gave values for roughness and profile height (Ra and $\mathrm{Rz}$ ): extreme values were obtained for the chemically and mechanically treated samples. Next, a pristine PA6 sample was used to compare the influence of the CNT content on the electric behavior of the samples. By increasing the pressure on the specimens, the volume resistivity decreased exponentially, while the surface resistivity showed no significant changes, independently of the CNT content. The obtained behavior proves a great potential of the MWNT-PA6 reinforced nanocomposites for sensor applications.
\end{abstract}

\section{Introduction}

Fullerene, which is a third allotropy form of carbon, was discovered in 1985, after the other known forms of carbon: graphite and diamond (which are hybridized sp2 and sp3, resp.). The most famous of all carbon forms in the fields of nanoscience are carbon nanotubes (CNTs). They were observed for the first time in 1991 by Sumio Iijima, a Japanese scientist, who used a high resolution transmission electron microscope (HRTEM). Carbon nanotubes can be found in two structures: single- or multiwalled (SWNT/MWNT) [1]. CNTs possess magnificent thermal, electrical, mechanical, and electrical properties which make them excellent candidates for a lot of applications. It is well known that CNTs can carry a current density of $10^{9} \mathrm{~A} / \mathrm{cm}^{2}$, which is the highest of any known materials $[2,3]$.
In order for CNT to be used effectively for obtaining polymer nanocomposites, an appropriate interfacial adhesion between the CNTs and the polymer matrix must be guaranteed [4-6]. There are usually two distinct techniques: mechanical and chemical. The mechanical approach consists of procedures such as ultrasonication, high shear mixing, and ball milling, while the chemical approach uses surface functionalization of CNTs, in order to improve their chemical compatibility to the polymer matrix and reduce their tendency to agglomerate. In the case of the chemical treatment, boiling acids are used $\left(\mathrm{H}_{2} \mathrm{SO}_{4}+\mathrm{HNO}_{3}\right)$ under ultrasonication, followed by boiling [4].

In this study, three distinct techniques were applied to the CNTs: chemical, mechanical, and mixed treatments. The last is a combination of the chemical and the mechanical treatments. 


\section{Experimental}

2.1. Materials. The materials used for this experiment are polyamide 6 (PA6), presented as pellets of $3 \mathrm{~mm}$ in diameter, bought from Sigma-Aldrich (France), with a molecular weight $M_{w}=150000 \mathrm{~g} \cdot \mathrm{mol}^{-1}$; nitric acid $\left(\mathrm{HNO}_{3}\right)$, purchased from Fisher Scientific (France); sulphuric acid $\left(\mathrm{H}_{2} \mathrm{SO}_{4}\right)$, $50 \%$ purity, also purchased from Fischer Scientific (France); 93\% purity multiwalled carbon nanotubes, with an external diameter of $11 \mathrm{~nm}$ and $3.2 \pm 1 \mathrm{~nm}$ thickness, purchased from Arkema (France). The sulphuric acid is a highly corrosive strong mineral acid, colourless to slightly yellow, viscous, and soluble in water at all concentrations. The nitric acid is also a highly corrosive acid, colourless, with a usual concentration of $68 \%$. Both acids are very toxic.

2.2. Protocol of Work. Firstly, three different types of treatments were applied to the MWNTs: chemical, mechanical, and mixed. The MWNT content was different as follows: $0.5 \%, 1 \%, 1.5 \%$, and $2 \%$. The three treatments are described below.

2.2.1. Chemical Treatment. A mixture of $65 \% \mathrm{HNO}_{3}$ and $50 \%$ $\mathrm{H}_{2} \mathrm{SO}_{4}(\mathrm{v} / \mathrm{v}=1: 3)$ was prepared in an Erlenmeyer glass and then $4 \mathrm{~g}$ of MWNTs was added. The solution was sonicated at $50^{\circ} \mathrm{C}$ for 4 hours. Then the MWNTs were separated from the mixture of acids and purified with oxygenated water until the $\mathrm{pH}$ value reached 7. Then the MWNTs were left for 48 hours into an oven at $130^{\circ} \mathrm{C}$, for drying. After the drying process, the MWNTs were weighted and there were $2.8 \mathrm{~g}$ left. The rest of $1.2 \mathrm{~g}$ was wasted during the washing procedure. Next, the acidic MWNTs were split into 4 different concentrations, $0.5 \%, 1 \%, 1.5 \%$, and $2 \%$, and dispersed into $27.77 \mathrm{~mL}$ of $90 \%$ $\mathrm{CH}_{2} \mathrm{O}_{2}$ (which is the equivalent of $25 \mathrm{~mL}$ of pure $\mathrm{CH}_{2} \mathrm{O}_{2}$ ) together with the PA6 pellets. The quantity of PA6 for each solution was calculated so that the final solutions should have a 20\% PA6 content. The solutions were left for 72 hours at $70^{\circ} \mathrm{C}$ on a magnetic stirrer in order to ease the dissolving process of the PA6 and homogenize the solution.

Four chemically treated solutions resulted. They were left to reach room temperature.

2.2.2. Mechanical Treatment. The first stage of the mechanical treatment was the separation of $0.1919 \mathrm{~g}, 0.3861 \mathrm{~g}, 0.5828 \mathrm{~g}$, and $0.7821 \mathrm{~g}$ of MWNT corresponding to the $0.5 \%, 1 \%$, $1.5 \%$, and $2 \%$ MWNT concentrations, into 4 different bottles. $27.77 \mathrm{~mL}$ of $90 \% \mathrm{CH}_{2} \mathrm{O}_{2}$ each was added and left for 1 hour for sonication at $50^{\circ} \mathrm{C}$. The process was followed by high mixing using the Ika T25 digital Ultra Turrax, at $18.000 \mathrm{rpm}$ for 30 minutes. The final step was adding the PA6 pellets quantity so that the final solution should have a $20 \%$ PA6 content and then they were left for 72 hours at $70^{\circ} \mathrm{C}$ on a magnetic stirrer. The 4 mechanically treated solutions which resulted were left to reach room temperature.

2.2.3. Mixed Treatment. The same mixture of $65 \% \mathrm{HNO}_{3}$ and $50 \% \mathrm{H}_{2} \mathrm{SO}_{4}(\mathrm{v} / \mathrm{v}=1: 3)$ which was used for the chemical treatment was prepared in an Erlenmeyer glass and $4 \mathrm{~g}$ of
MWNTs was added. It was left for sonication at $50^{\circ} \mathrm{C}$ for 4 hours and then mixed with Ultra Turrax at $18.000 \mathrm{rpm}$ for 30 minutes. Then the MWNTs were separated from the mixture of acids and washed with oxygenated water until the $\mathrm{pH}$ value reached 7. Next, the MWNTs were put into an oven at $130^{\circ} \mathrm{C}$, for 48 hours, for drying. Similar to the previous two procedures, the mixed treated MWNTs were split into 4 different concentrations, $0.5 \%, 1 \%, 1.5 \%$, and $2 \%$, and then dispersed into $27.77 \mathrm{~mL}$ of $90 \% \mathrm{CH}_{2} \mathrm{O}_{2}$ together with the PA6 pellets. The solutions were left for 72 hours at $70^{\circ} \mathrm{C}$ on a magnetic stirrer. Four mixed treated solutions resulted. They were left to reach room temperature.

2.2.4. Electrospinning Conditions. For the electrospinning procedure, an experimental device, built in the "Laboratoire de Physique et Mécanique Textiles," ENSISA, Mulhouse (France), was used [7]. The 12 obtained solutions were electrospun for 15 minutes at $30 \mathrm{kV}$ voltage, room conditions of $30 \%$ humidity, and $20^{\circ} \mathrm{C}$. A $0.7 \times 30 \mathrm{~mm}$ needle was used, with a $15 \mathrm{~cm}$ distance from the needle to the collecting area and a $0.283 \mathrm{~mL}$ feed rate was applied.

2.2.5. Structural Analysis of the Samples. The obtained samples have been characterized using the scanning electron microscopy (SEM, Hitachi S-2360N) and the atomic force microscopy (AFM, NTEGRA Spectra NT-MDT). Table 1 shows the SEM results, for each CNT concentration. In these pictures, 100 different fibers of each sample were measured in order to evaluate their diameters.

Table 3 shows the AFM results for each CNT concentration. The surface roughness for 100 nanofibers per sample was calculated using the Gwyddion free software. Average values of the roughness $(\mathrm{Ra})$ and the average profile height $(\mathrm{Rz})$ were obtained (Table 2).

The extreme $\mathrm{Ra}$ and $\mathrm{Rz}$ values were obtained for the chemically and mechanically treated samples:

(i) $\min : 0.5 \%$ CNT content, chemical treatment: $\mathrm{Ra}=$ $2 \mathrm{~nm} ; \mathrm{Rz}=9 \mathrm{~nm}$;

(ii) $\max : 0.5 \% \mathrm{CNT}$ content, mechanical treatment: $\mathrm{Ra}=$ $11.2 \mathrm{~nm} ; \mathrm{Rz}=57.8 \mathrm{~nm}$.

2.2.6. Preparing the Samples for the Resistance Measurements. In order to measure the volume resistance, three specimens of $1 \times 1 \mathrm{~cm}$ were cut from each of the obtained samples, while for the surface resistance the cut dimension was $1 \times 3 \mathrm{~cm}$. Both the surface and the volume resistance were measured up to the American Standard ASTM D 257-61, using homemade plate electrodes. The electrodes were made from copper and they were previously metallized with a gold layer. Before the measuring procedure, the samples were left for 24 hours in the room where they were to be tested, in atmospheric conditions of $20 \pm 2^{\circ} \mathrm{C}$ and $60 \pm 2 \%$ humidity. The device used was the Knick Terra Ohm Meter (LPMT, ENSISA, Mulhouse, France).

An assembly of loads with the purpose of studying the effect of compression on resistivity was also used (Figure 1).

Table 4 contains the parameters of the electrical resistance measurements. 
TABLE 1: SEM results.

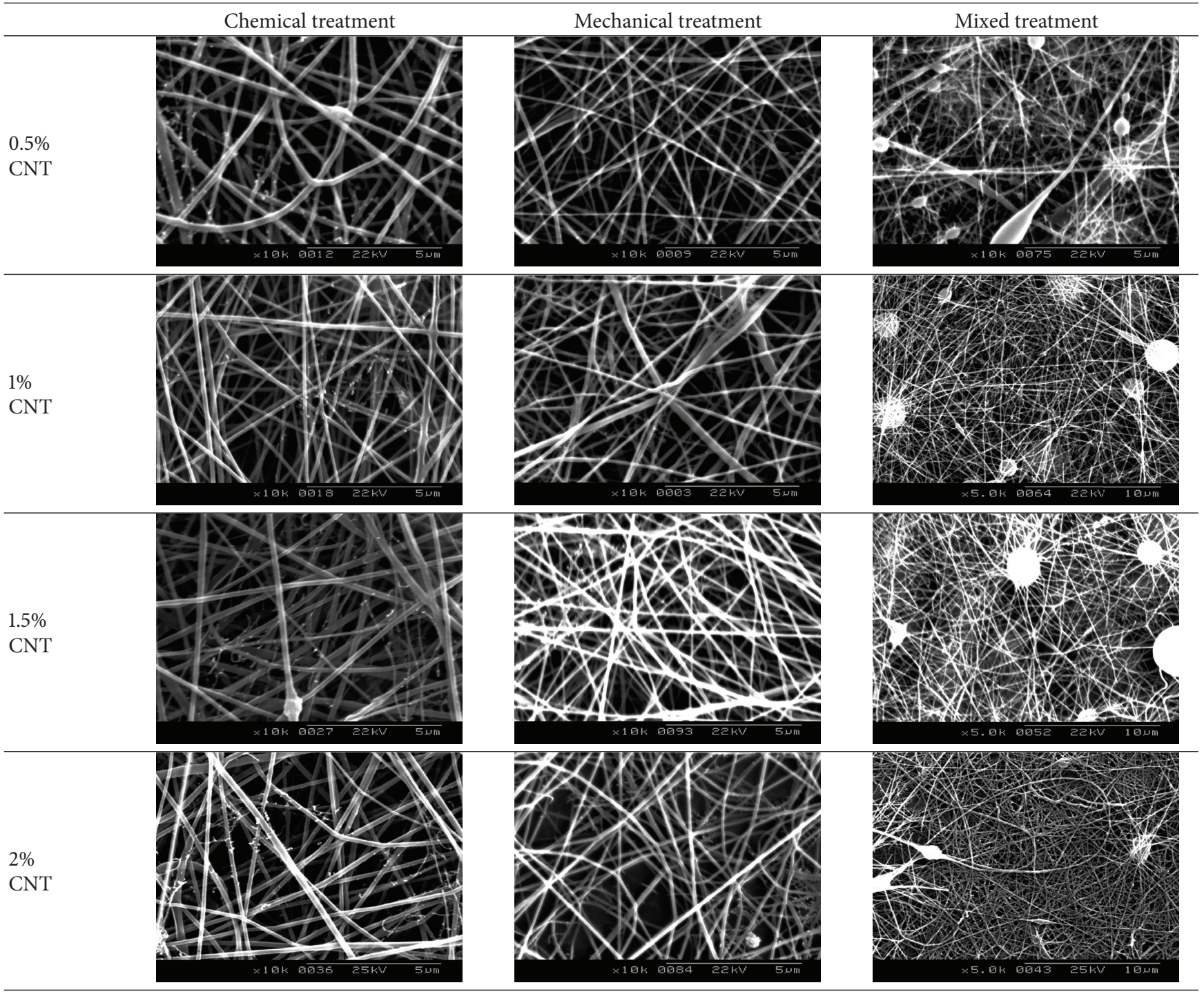

TABLE 2: Average values of the roughness $(\mathrm{Ra})$ and profile height $(\mathrm{Rz})$ for the electrospun nanofibers.

\begin{tabular}{|c|c|c|c|c|c|c|c|c|c|c|c|c|}
\hline & \multicolumn{4}{|c|}{ Chemical treatment } & \multicolumn{4}{|c|}{ Mechanical treatment } & \multicolumn{4}{|c|}{ Mixed treatment } \\
\hline & $0.5 \%$ & $1 \%$ & $1.5 \%$ & $2 \%$ & $0.5 \%$ & $1 \%$ & $1.5 \%$ & $2 \%$ & $0.5 \%$ & $1 \%$ & $1.5 \%$ & $2 \%$ \\
\hline $\begin{array}{l}\mathrm{Ra} \\
(\mathrm{nm})\end{array}$ & 2 & 3.8 & 7 & 6.4 & 11.2 & 2.42 & 2.24 & 3.2 & 9 & 4.5 & 4.68 & 5.2 \\
\hline $\begin{array}{l}\mathrm{Rz} \\
(\mathrm{nm}) \\
\end{array}$ & 9 & 20.2 & 32.6 & 27.2 & 57.8 & 10.36 & 13.4 & 14.2 & 23 & 19.56 & 17.44 & 20.2 \\
\hline
\end{tabular}

\section{Results and Discussions}

3.1. The Influence of the CNT Content on the Morphology of the Nanofibers. For the SEM analysis, for each sample, specimens from 3 different areas were taken: two extremities and the center. The diameters of the nanofibers varied from $43.37 \mathrm{~nm}$ corresponding to the $0.5 \%$ CNT mixed treated sample to $304.71 \mathrm{~nm}$ corresponding to the $0.5 \% \mathrm{CNT}$ chemically treated sample. Analysing the values of the obtained diameters, the obtained nanofibers show a high level of homogeneity. As far as defects are concerned, the chemically treated samples exhibit solvent traces on the extremity specimens, which caused that the nanofibers stick to each other. At the same time, the nanofibers from the extremities are not uniform and are flat. However, the nanofibers analysed from the center specimens have a uniform aspect with a homogenous structure. Occasionally, few beads are also present [8].

In the case of mechanically treated samples, the traces of solvent are significantly reduced if compared to 

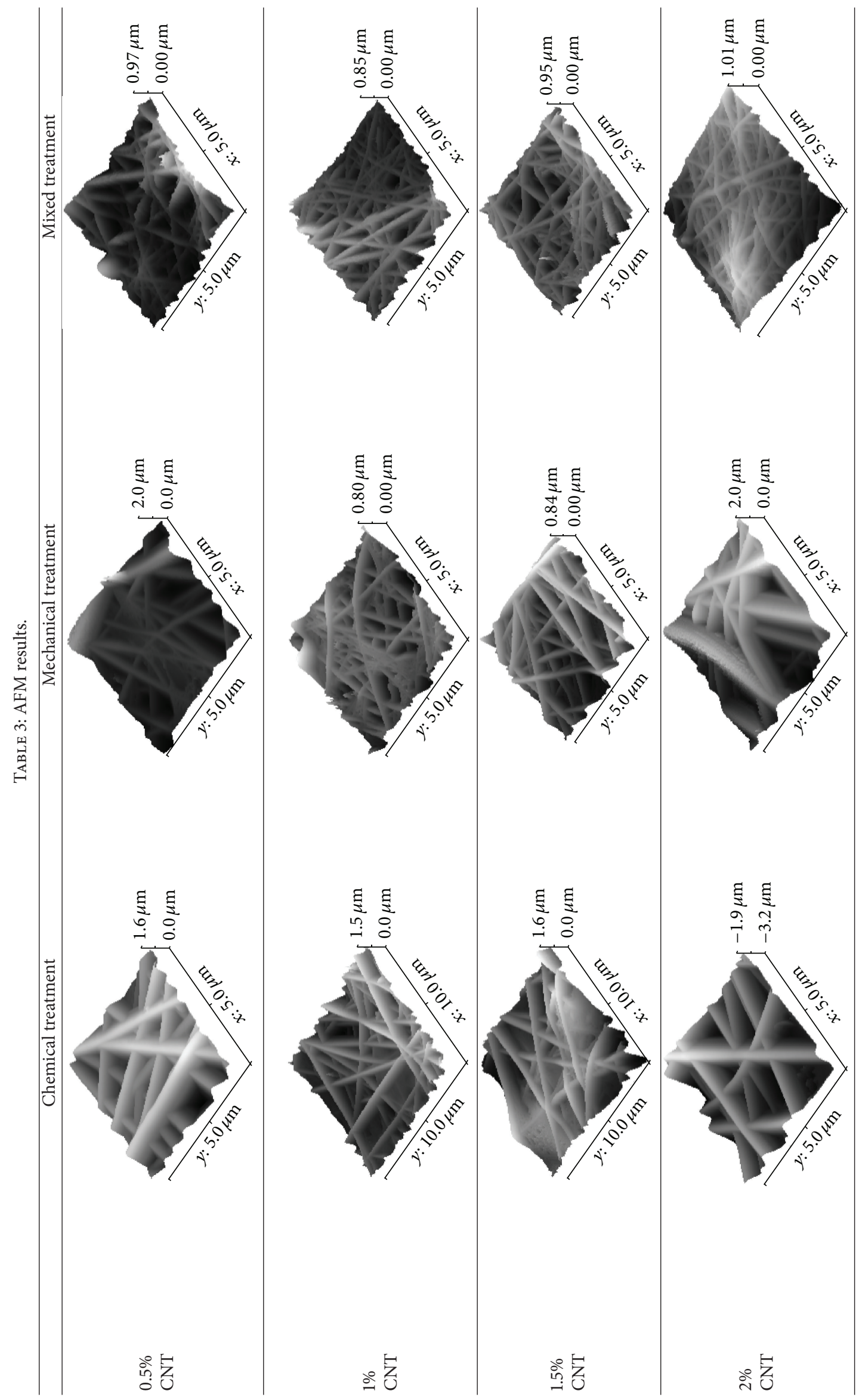


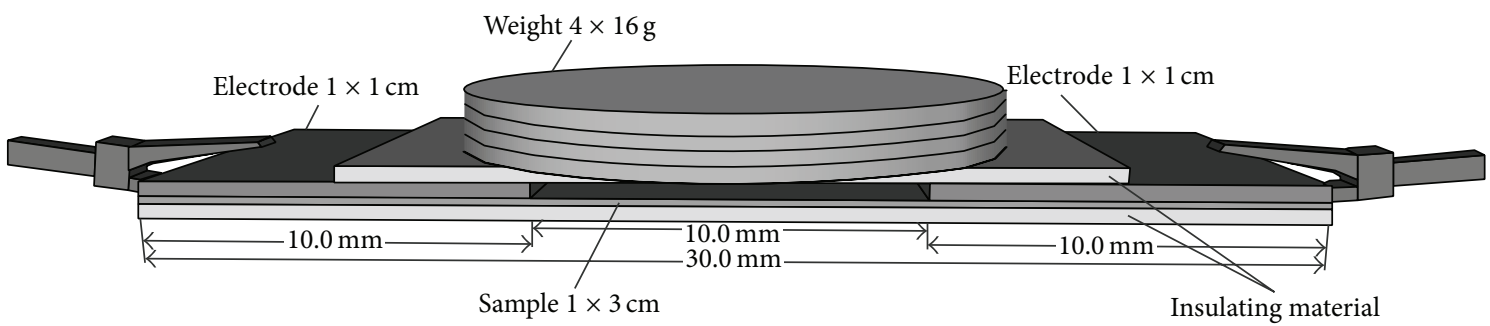

(a)

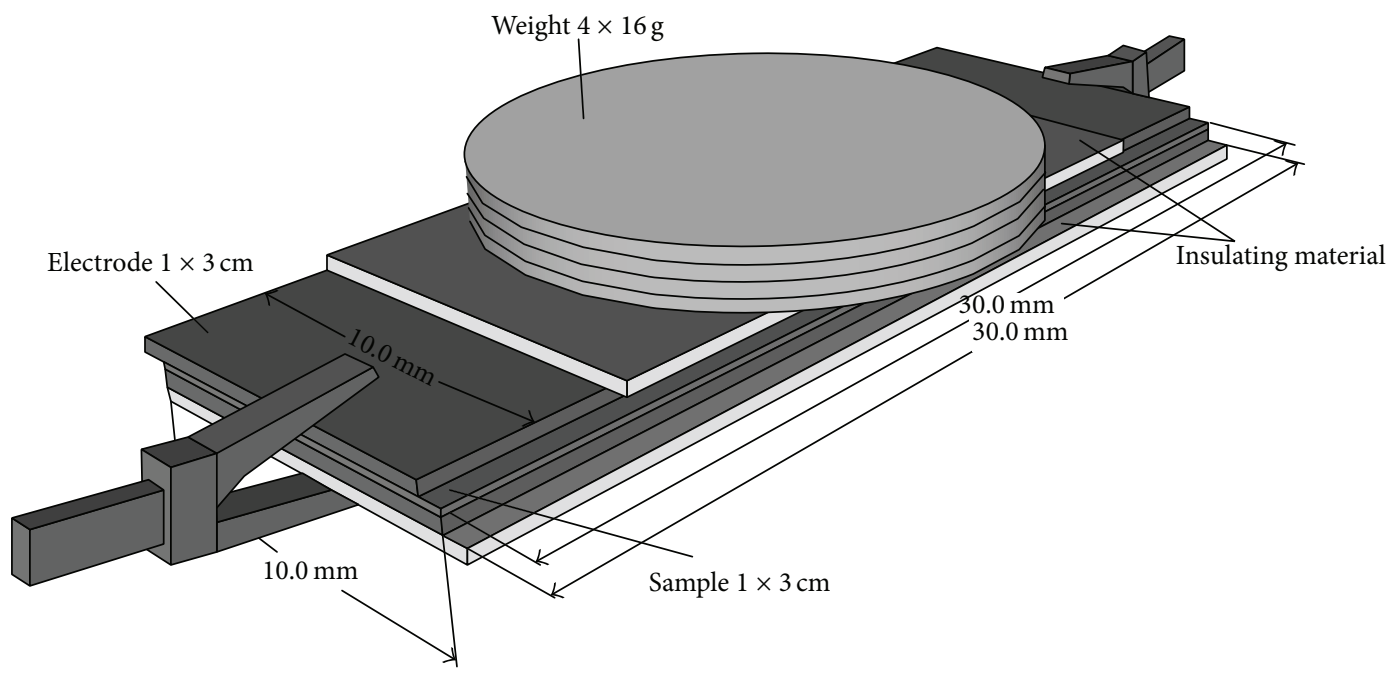

(b)

FIGURE 1: Surface resistance setup versus volume resistance setup.

TABLE 4: Parameters of the electrical resistance measurements.

\begin{tabular}{lcc}
\hline Resistance type & Surface & Volume \\
\hline Dimension of the specimens & $1 \times 1 \mathrm{~cm}$ & $1 \times 3 \mathrm{~cm}$ \\
\hline
\end{tabular}

(i) No cleaning

Conditioning of the specimen

(ii) No predrying

(iii) $24 \mathrm{~h}$ of conditioning

Atmospheric testing conditions $20 \pm 2^{\circ} \mathrm{C}$

$60 \pm 2 \% \mathrm{RH}$

\begin{tabular}{lcc}
\hline Applied voltage & $500 \mathrm{~V}$ & $100 \mathrm{~V}$ \\
\hline Time of electrification & \multicolumn{3}{c}{$2 \mathrm{~min}$} \\
\hline
\end{tabular}

the chemically treated ones. Instead, the number of beads is higher. Another important aspect is that the electrospinning jet was not continuous, as droplets of solution are present on the surface of the nanofibers.

The mixed treatment led to the following results: very high number of beads on the surface of the nanofibers and flat fibers. This is caused by the fact that the CNT structure was influenced by two important factors: chemical and mechanical treatments. It can be concluded that both treatments combined proved to be too aggressive for the $\mathrm{CNT}$ structure.
Next we will analyse the percolation threshold of the nanocomposites and the influence of the CNT on the volume resistivity of the chemically treated nanofibers.

\subsection{Percolation Threshold of the Reinforced Nanocomposites} and the Influence of the CNT Content on the Volume Resistivity of the Nanocomposites. Figure 2 shows the behaviour of the electrical volume conductivity in $\mathrm{S} / \mathrm{m}$ of the pristine PA6 sample, compared to the reinforced PA6, at different CNT content, for each of the 3 applied treatments. The obtained results have shown that the percolation threshold is below the $0.5 \%$ concentration. Increasing the CNT, conductivity also increases, which proves that the electrical status of the nanocomposite changes from an insulative into static dissipative material (where the conductivity is between $1 \times$ $10^{-4} \mathrm{~S}$ and $\left.1 \times 10^{-11}\right)[9-11]$.

One can also notice that the values of the volume resistivity of the samples decrease together with the applied load (Figure 3). This shows the fact that the nanocomposites can be used as pressure sensors and be integrated in smart textiles [12].

3.3. The Influence of the CNT Content on the Surface Resistivity of Nanocomposites. Due to the fact that the dimension of the analysed specimens was $1 \times 3 \mathrm{~cm}$ and the dimension of the electrodes was $1 \times 1 \mathrm{~cm}$, the distance between the electrodes equals the dimension of the contact surface $(1 \times 1 \mathrm{~cm})$. 


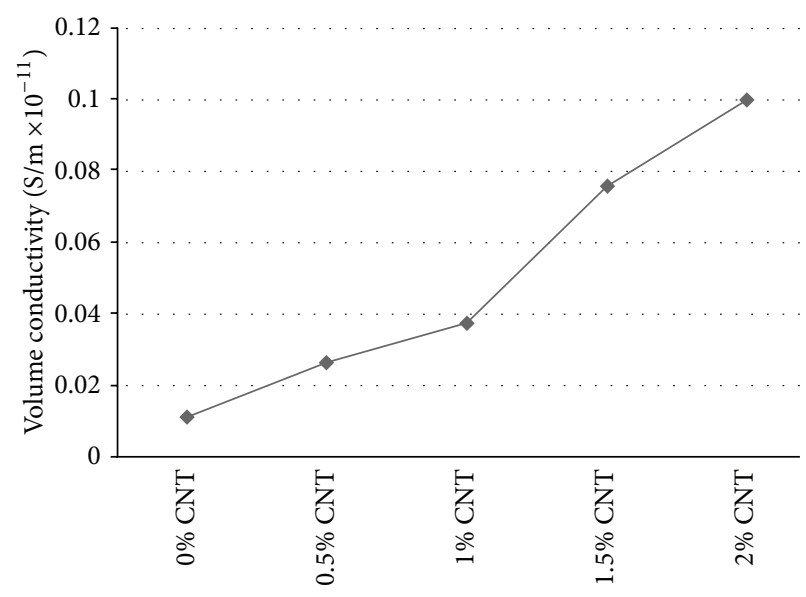

(a)

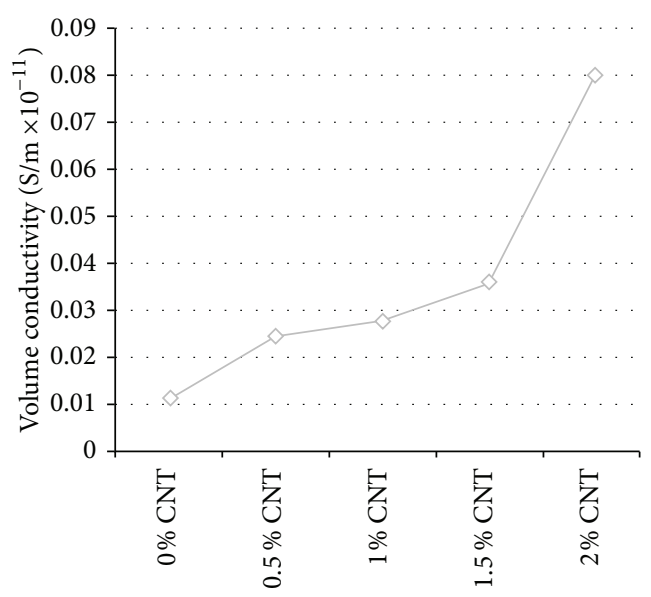

(b)

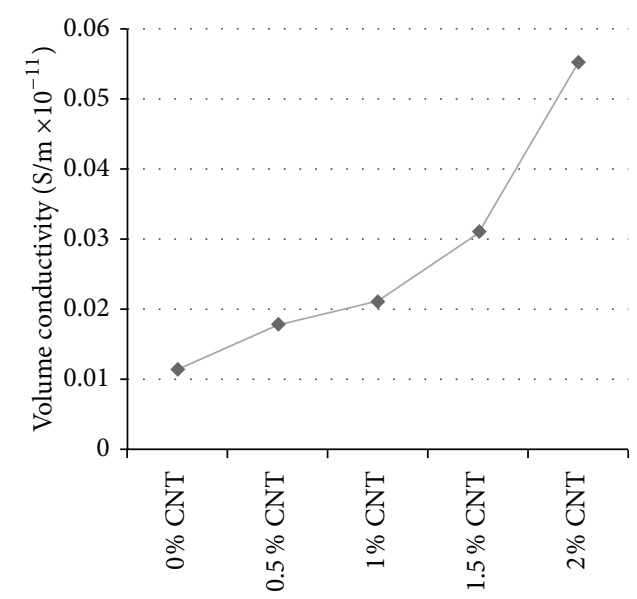

(c)

Figure 2: Volume conductivity of the $20 \%$ CNT PA6 composite with different CNT content: (a) chemical treatment; (b) mechanical treatment; (c) mixed treatment.

The CNTs are present both inside and on the surface of the nanofibers [13]. This means that the values of the surface resistivity are the same with those of the surface electrical resistance.

Figure 4 shows the evolution of the surface electrical resistivity for the obtained samples. There were no significant changes for the surface resistivity of the samples. Neither the CNT content nor the applied treatment has shown a significant change in the surface resistivity in relation to the applied load.

We note that the electrical behaviour of the PA6-CNT nanocomposites analysed in the current paper (volume resistivity and conductivity, surface resistivity and conductivity) can be theoretically approached using the two nanocomposite models, (the Tanaka or the Tsagaropoulos model) $[14,15]$. In such a context, different dependencies of the above mentioned electrical parameters can be accepted (temperature dependency, etc.) so that the dynamics analysis can become more complex but complete. We consider that this study requires a separate analysis which will be done in a future paper.

\section{Conclusions}

The main conclusions of the present paper are as follows:

(i) as far as defects are concerned, the chemically treated solutions gave the most uniform nanofibers;

(ii) the biggest number of defects found on the nanofibers resulted from the mixed treatment;

(iii) the percolation threshold could not be reached; it is below $0.5 \%$ concentration;

(iv) increasing the $\mathrm{CNT}$, the volume conductivity also increases;

(v) the values of the volume resistivity of the samples decrease together with the applied load;

(vi) for surface resistivity, no significant changes were observed; neither the CNT content nor the applied treatment has shown a significant change in the surface resistivity in relation to the applied load. 


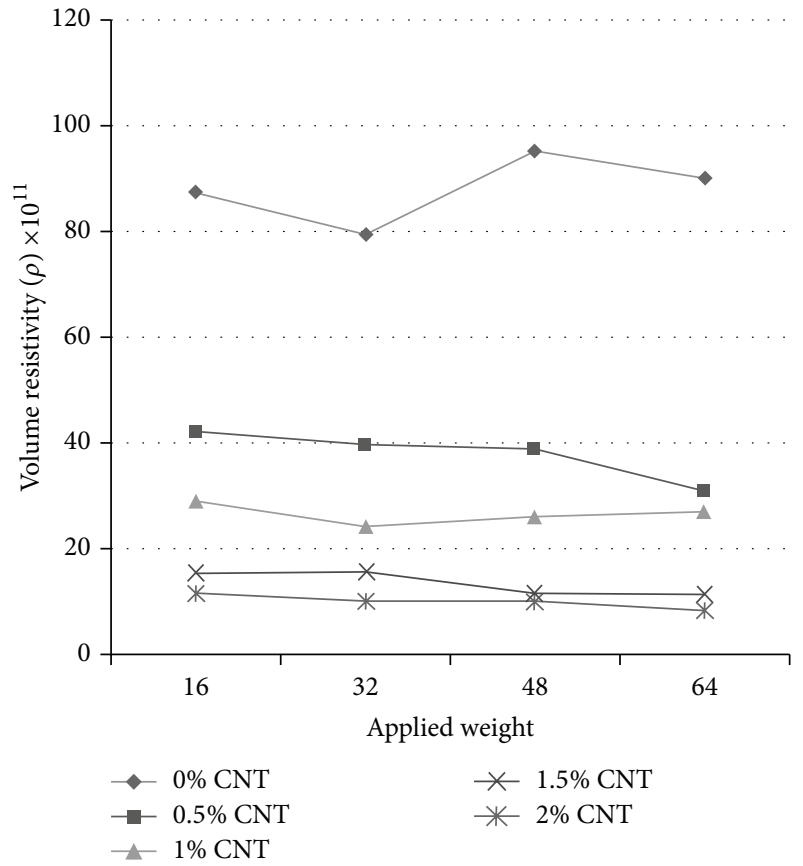

(a)

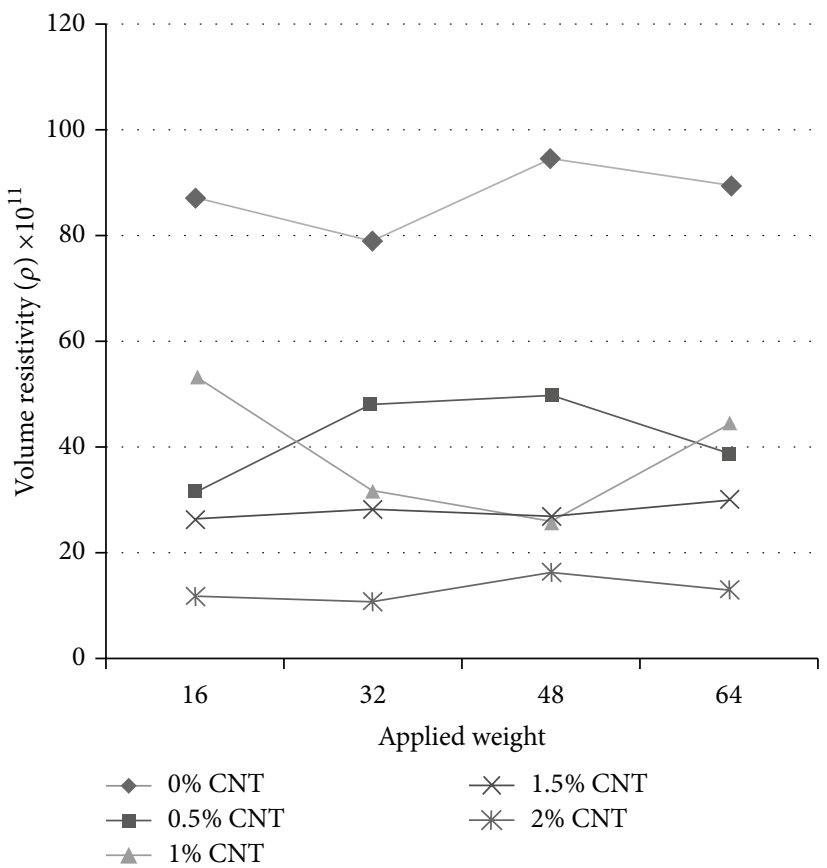

(b)

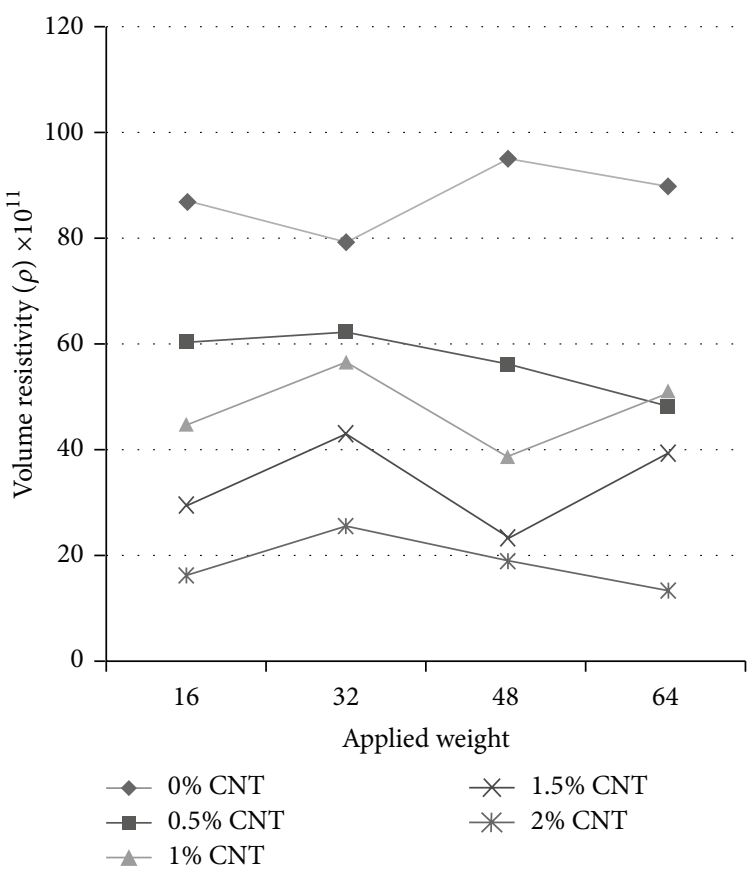

(c)

FIGURE 3: Volume resistivity of the 20\% CNT PA6 composite with different CNT content: (a) chemical treatment; (b) mechanical treatment; (c) mixed treatment.

\section{Conflict of Interests}

The authors declare that there is no conflict of interests.

\section{Authors' Contribution}

M. A. Călin created the experimental solutions; electrospun the solutions; was responsible for SEM analysis of the nanofibers, AFM analysis of the nanofibers, analysis of the volume behavior of the nanofibers, analysis of the surface resistivity behavior of the nanofibers, and interpretation of the results; and has written the paper. L. R. Manea has helped with the interpretation of the electrospinning parameters. L. Schacher has provided support and assistance for the measurements and has provided the equipment required for the experimental research. D. Adolphe has provided support 


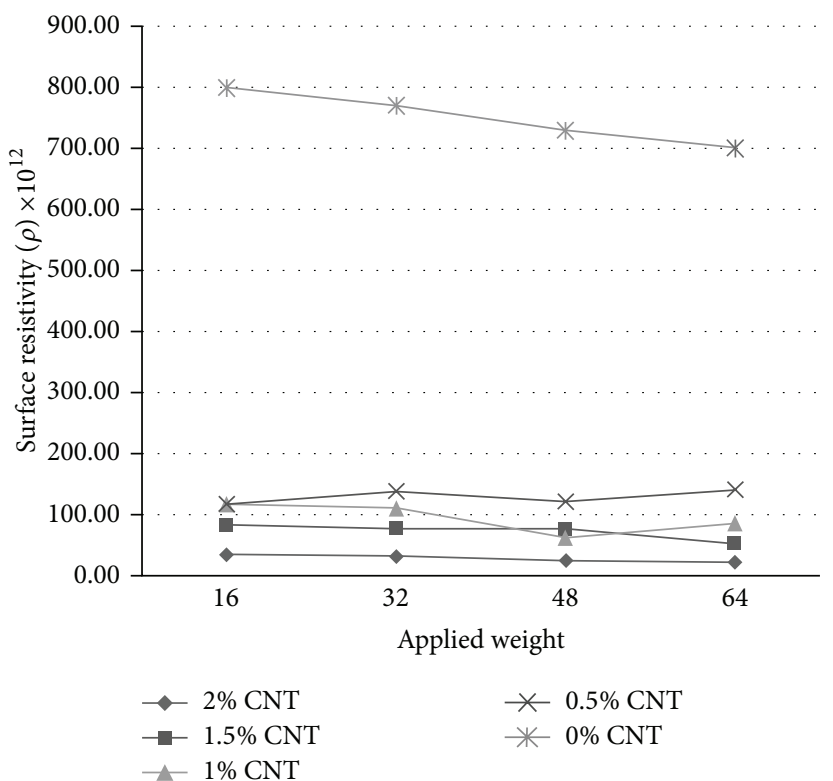

(a)

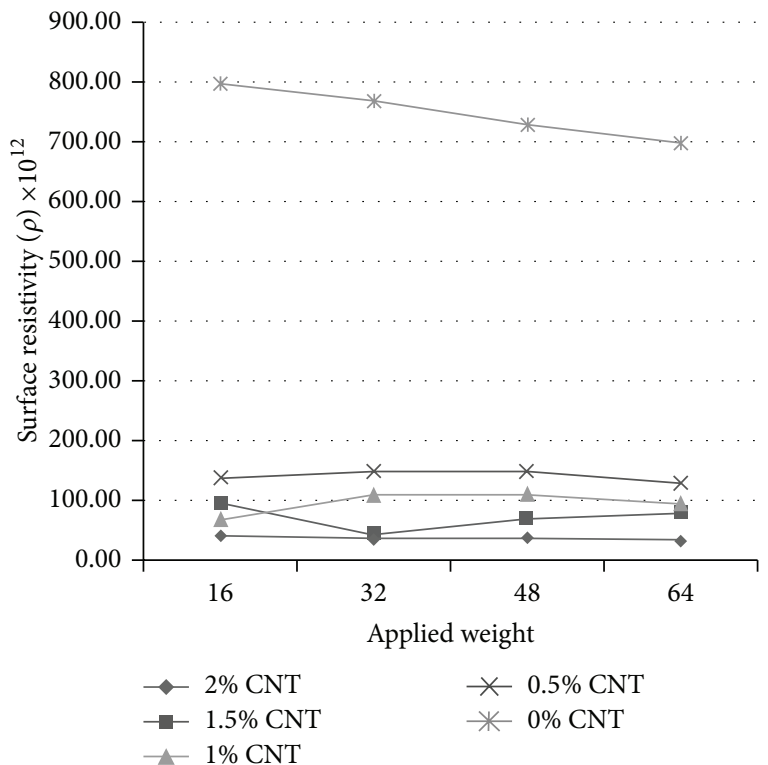

(b)

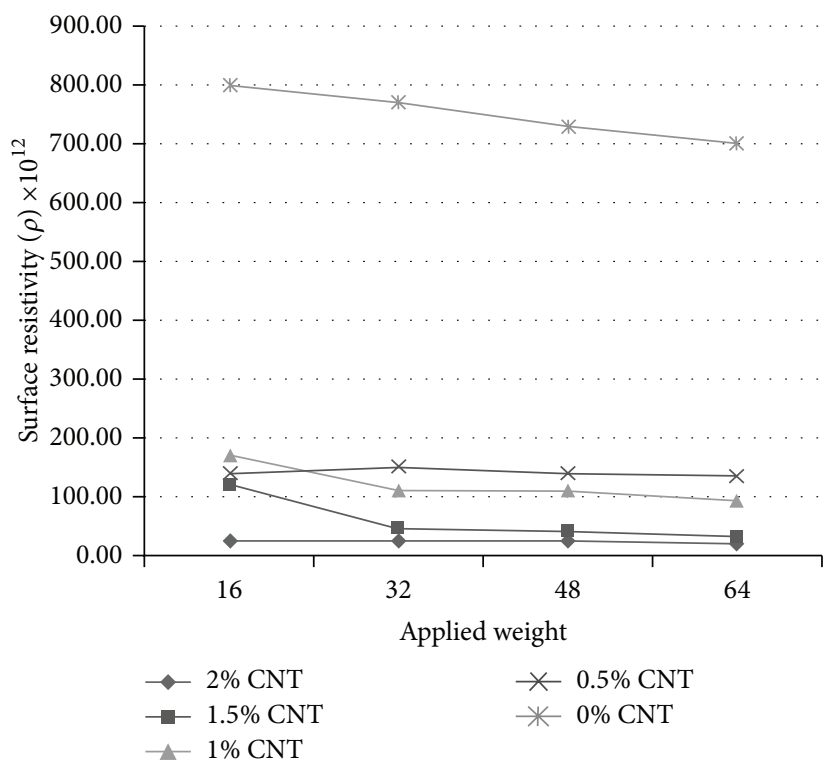

(c)

FIGURE 4: Surface resistivity of the $20 \%$ CNT PA6 composite with different CNT content: (a) chemical treatment; (b) mechanical treatment; (c) mixed treatment.

and assistance for the measurements and has provided the equipment required for the experimental research. A. L. Leon has helped with the interpretation of the electrospinning parameters. G. L. Potop has helped with the interpretation of the electrospinning parameters. M. Agop coordinated the interpretation and analysis of the electrical results.

\section{References}

[1] M. A. Călin, S. Almuhamed, N. Khenoussi et al., "Characterization of MWNT-reinforced PA6 nanocomposites (produced by electrospinning)," in Proceedings of the 12th World Conference Autex, Zadar, Croatia, June 2012.
[2] B. Q. Wei, R. Vajtai, and P. M. Ajayan, "Reliability and current carrying capacity of carbon nanotubes," Applied Physics Letters, vol. 79, no. 8, pp. 1172-1174, 2001.

[3] A. P. S. Sawhney, B. Condon, K. V. Singh, S. S. Pang, G. Li, and D. Hui, "Modern applications of nanotechnology in textiles," Textile Research Journal, vol. 78, no. 8, pp. 731-739, 2008.

[4] P.-C. Ma, N. A. Siddiqui, G. Marom, and J.-K. Kim, "Dispersion and functionalization of carbon nanotubes for polymer-based nanocomposites: a review," Composites Part A: Applied Science and Manufacturing, vol. 41, no. 10, pp. 1345-1367, 2010.

[5] X. L. Xie, Y.-W. Mai, and X.-P. Zhou, "Dispersion and alignment of carbon nanotubes in polymer matrix: a review," Materials Science and Engineering R: Reports, vol. 49, no. 4, pp. 89-112, 2005. 
[6] B. Fiedler, F. H. Gojny, M. H. G. Wichmann, M. C. M. Nolte, and K. Schulte, "Fundamental aspects of nano-reinforced composites," Composites Science and Technology, vol. 66, no. 16, pp. 3115-3125, 2006.

[7] N. Khenoussi, L. Schacher, and D. C. Adolphe, "Nanofiber production: study and development of electrospinning device," Experimental Techniques, vol. 36, no. 2, pp. 32-39, 2012.

[8] Y. Liu, J.-H. He, J.-Y. Yu, and H.-M. Zeng, "Controlling numbers and sizes of beads in electrospun nanofibers," Polymer International, vol. 57, no. 4, pp. 632-636, 2008.

[9] S. Almuhamed, N. Khenoussi, L. Schacher, D. Adolphe, and H. Balard, "Measuring of electrical properties of MWNTreinforced PAN nanocomposites," Journal of Nanomaterials, vol. 2012, Article ID 750698, 7 pages, 2012.

[10] S. Pfeifer, S.-H. Park, and P. R. Bandaru, "Analysis of electrical percolation thresholds in carbon nanotube networks using the Weibull probability distribution," Journal of Applied Physics, vol. 108, no. 2, Article ID 024305, 2010.

[11] J. Li, P. C. Ma, C. W. Sze, T. C. Kai, B. Z. Tang, and J.-K. Kim, "Percolation threshold of polymer nanocomposites containing graphite nanoplatelets and carbon nanotubes," in Proceedings of the 16th International Conference on Composite Materials (ICCM '07), July 2007.

[12] ESDA, ESD Association Advisory for Electrostatic Discharge Terminology, ESD Association, 2009.

[13] J. M. Deitzel, J. Kleinmeyer, D. Harris, and N. C. B. Tan, “The effect of processing variables on the morphology of electrospun nanofibers and textiles," Polymer, vol. 42, no. 1, pp. 261-272, 2001.

[14] M. G. Danikas, "On two nanocomposite models: differences, similarities and interpretational possibilities regarding Tsagaropoulos' model and Tanaka's model," Journal of Electrical Engineering, vol. 61, no. 4, pp. 241-246, 2010.

[15] M. G. Danikas, A. Bairaktari, R. Sarathi, and A. Basri Bin Abd Ghani, "A review of two nanocomposite insulating materials models: Lewis' contribution in the development of the models, their differences, their similarities and future challenges," Engineering, Technology \& Applied Science Research, vol. 4, no. 3, pp. 636-643, 2014. 

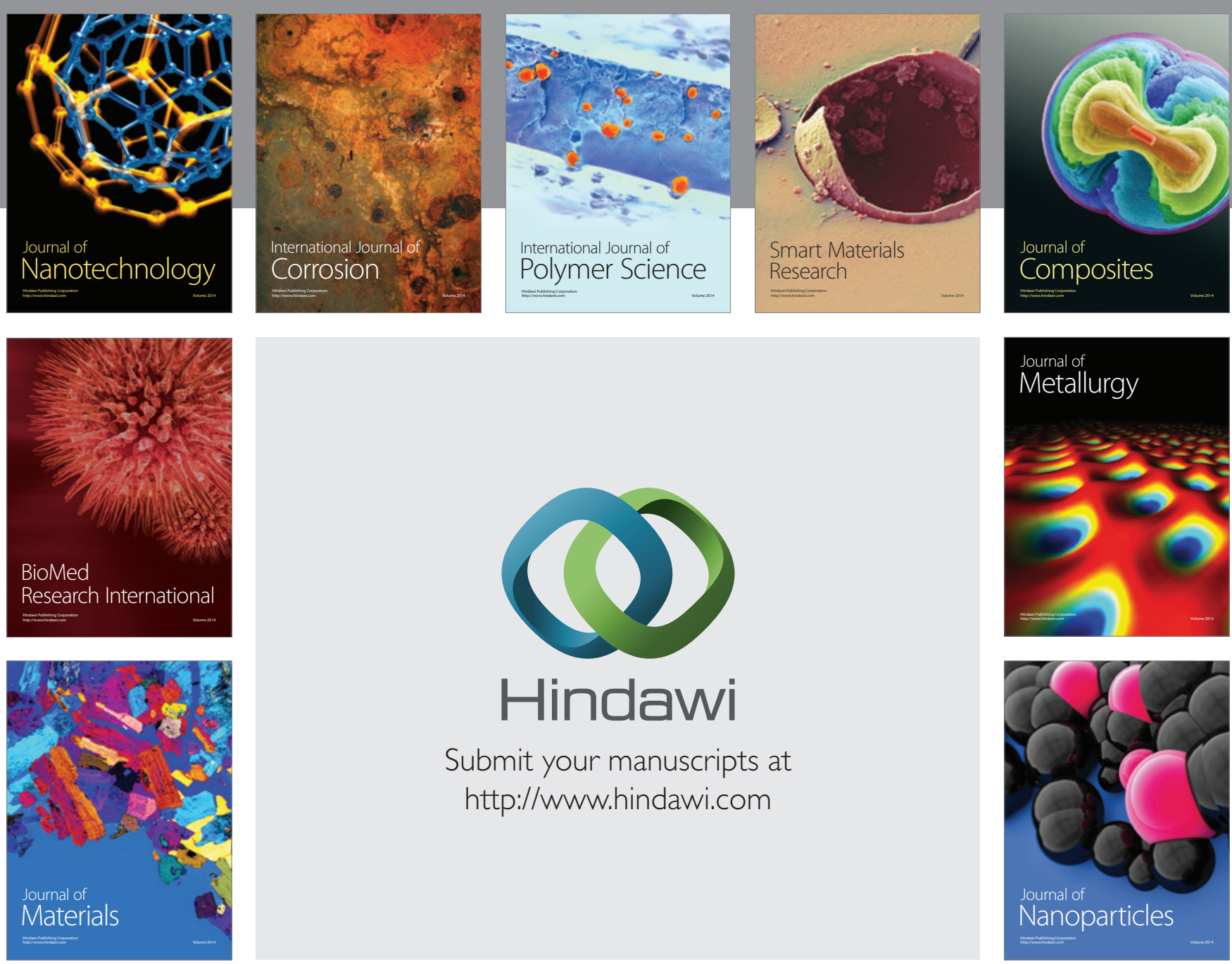

Submit your manuscripts at http://www.hindawi.com
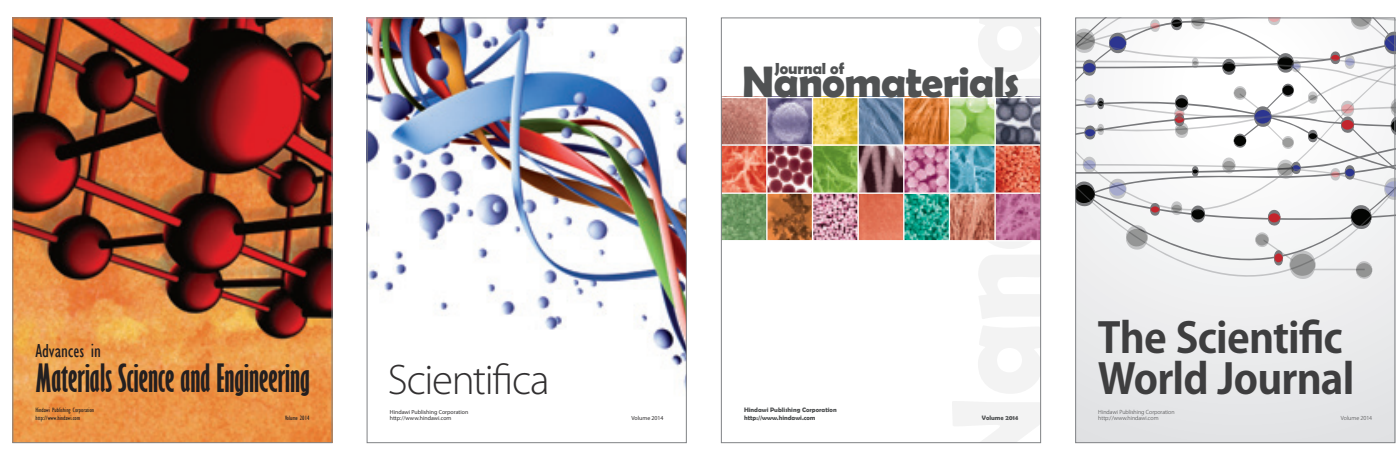

\section{The Scientific World Journal}
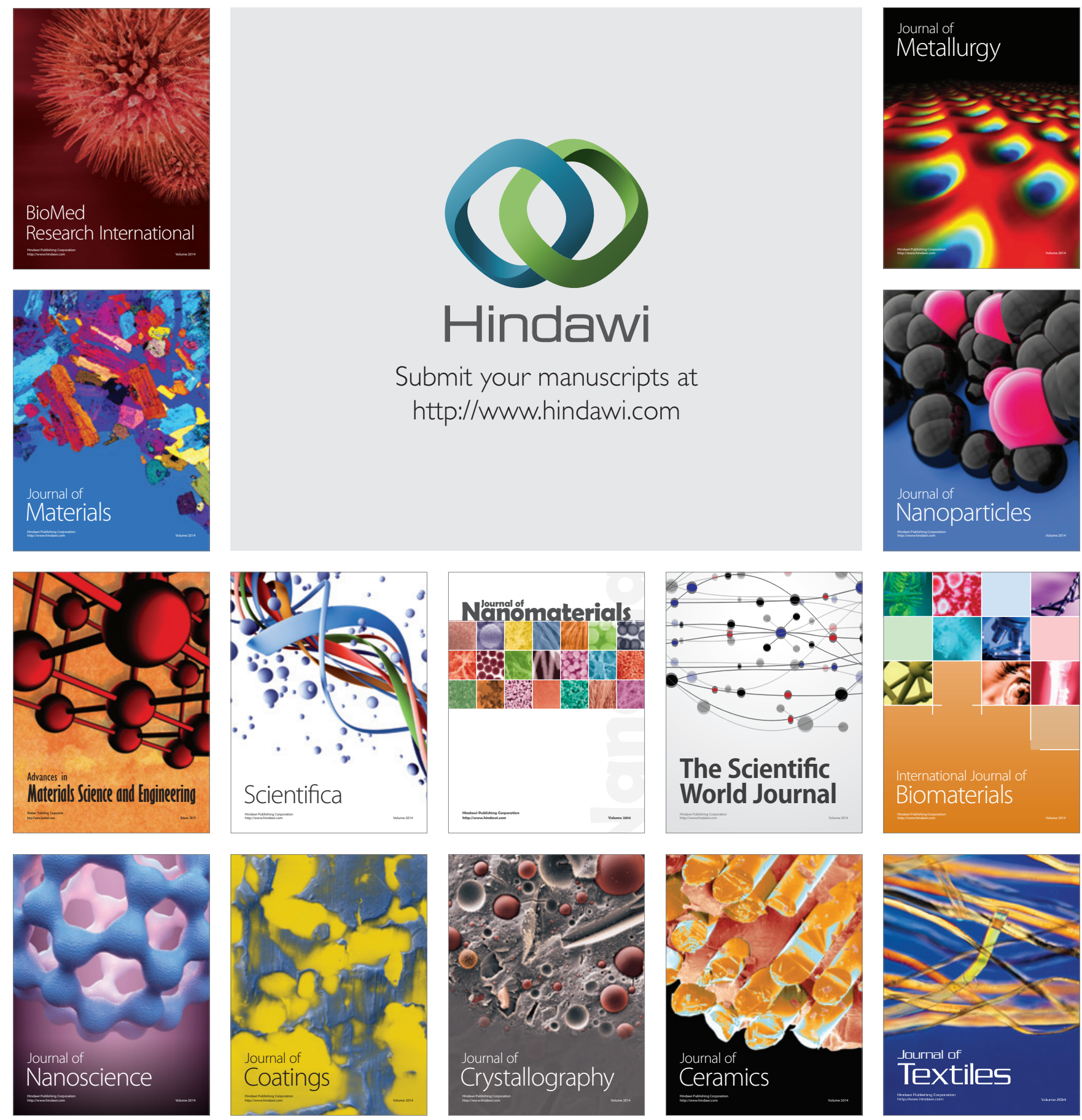